\title{
Histoire d'une absence : la place du père dans les œuvres des écrivaines chinoises des années 1920.
}

Jacqueline ESTRAN

\section{(2) OpenEdition}

\section{Journals}

Édition électronique

URL : http://journals.openedition.org/transtexts/496

DOI : $10.4000 /$ transtexts.496

ISSN : 2105-2549

Éditeur

Gregory B. Lee

Référence électronique

Jacqueline ESTRAN, « Histoire d'une absence : la place du père dans les œuvres des écrivaines chinoises des années 1920. », Transtext(e)s Transcultures 跨文本跨文化 [En ligne], 8| 2013, mis en ligne le 02 décembre 2013, consulté le 01 mai 2019. URL : http://journals.openedition.org/ transtexts/496 ; DOI : 10.4000/transtexts.496 


\title{
Transtext(e)s Transcultures 跨文本跨 文化
}

Journal of Global Cultural Studies

$8 \mid 2013$ :

Genre et filiation : pratiques et représentations

Pères, mères, n` urrices

\section{Histoire d'une absence : la place du père dans les œuvres des écrivaines chinoises des années 1920.}

\author{
JACQUELINE ESTRAN
}

\section{Résumés}

Français English

La filiation occupe, en tant que lien aux parents ou à de plus lointaines origines, une place incontournable dans la vie de tout individu et de toute société. Centrale dans l'organisation de la société traditionnelle chinoise, elle est fortement remise en cause au début du XXe siècle : révolte des fils contre leur père, révolte des filles contre la tradition. Et si les recherches se sont penchées sur la représentation de la mère, les références au père sont peu fréquentes. Les œuvres de quatre écrivaines représentatives, Bing Xin 冰心 (1900-1999), Lu Yin 盧隱 (1898-1934), Ling Shuhua 淩叔華 (1900-1990) et Feng Yuanjun 馮沅君 (1900-1974), sont interrogées ici quant à cette absence.

As a transgenerational bond, filiation occupies a main role in every individual's life as well as in every society. Central to the organization of Chinese traditional society, it is heavily questioned at the beginning of twentieth-century: revolt of sons against their father, revolt of girls against tradition. If researches have focused on the maternal figure in women's literature, far less is known about the father. The works of four representative women writers, Bing Xin 冰心 (1900-1999), Lu Yin 盧隱 (1898-1934), Ling Shuhua 淩叔華 (1900-1990) and Feng Yuanjun 馮沅君 (1900-1974), will be questioned on this lack of paternal figure in their writings. 


\section{Texte intégral}

1 La filiation occupe, en tant que lien aux parents ou à de plus lointaines origines, une place incontournable dans la vie de tout individu et de toute société. Centrale dans l'organisation de la société traditionnelle chinoise, elle est fortement remise en cause au début du XXe siècle : révolte des fils contre leur père, révolte des filles contre la tradition. Et si les recherches se sont penchées sur la représentation de la mère, vue comme présente ou présente par son absence ${ }^{1}$, les références au père sont peu fréquentes.

2 La Chine est une société en crise majeure dans les années 1910-1920 et, au-delà de la famille et de l'individu, la notion de filiation intervient dans un contexte historique, social et culturel qui sera interrogé afin d'éclaircir les enjeux en question. Seront abordées, ensuite, les œuvres de quatre écrivaines représentatives de la période ${ }^{2}$ afin de voir comment elles ont négocié ce rapport à la filiation et, ici, au père dans ce contexte très particulier des années 1920.

\section{Filiation et contexte culturel, social, politique}

3 La Chine de la première moitié du XXe siècle se caractérise par la rupture, une rupture qui intervient à tous les niveaux, du plus large - la société - au plus restreint - l'individu - et sur tous les plans : culturel, social, politique, avec toutes les subdivisions possibles.

4 Sur un plan politique, la rupture se traduit par un changement de régime : le passage, en 1912, d'une dynastie impériale à une république en quête de démocratie entraîne une remise en question des structures politiques existantes, connues ou à créer et, donc, des structures sociales qui en découlent. En Chine, il ne s'agit pas d'un simple changement de régime, le gouvernement ne contrôlant pas tout le territoire, mais d'expérimentations, de mise en pratique d'idées diverses et, au final, de la coexistence de différents systèmes sur une trentaine d'années. Il y a donc à la fois remise en question du passé mais aussi de ce qui est en train de se faire ou de ce qui pourrait se faire, ce qui engendre une grande instabilité du système de valeurs auquel les individus se réfèrent.

5 Dans le domaine littéraire, le passage de la langue classique (wenyan) à la langue vernaculaire (baihua) qui se fait sur un mode révolutionnaire, entre 1916 et 1919, entraîne une remise en question tant des objectifs de la littérature que des différents genres littéraires. Deux « tendances majeures » se dégagent alors: une critique de la structure familiale traditionnelle et une remise en cause des institutions morales, religieuses et légales qui entravent le développement de l'individu.

6 Ces ruptures s'enchaînent et s'imbriquent, engendrant au plan individuel une remise en question de tous les repères. Et, dans ce contexte presque paroxystique de ruptures, la filiation, en tant que lien, prend un sens particulier.

7 Comment donc, dans un tel contexte, la filiation a-t-elle été vécue, représentée?

8 Comme une rupture supplémentaire ? Et comment les écrivains négocient-ils 
cette nécessité de la rupture avec celle de la continuité filiale?

La période de référence est, ici, la plus radicale : les années 1920, celle de toutes les ruptures concommittantes.

\section{Filiation, écrits féminins et écrits masculins}

Si la distinction entre écrits féminins et masculins peut sembler, et est en général arbitraire, elle s'avère ici signifiante car, dans leur rapport à l'écrit, hommes et femmes prennent deux voies distinctes et, dans leur rapport filiaux, développent deux types de relations - spécifiques - au père et à la mère. ${ }^{3}$

$11 \mathrm{Si}$, dans leur rapport à l'écrit, tous commencent par la nouvelle, rapidement, les hommes se lancent dans l'écriture de romans là où les femmes privilégient des formes courtes, comme la nouvelle ou la prose littéraire ou des formes intimistes comme le genre épistolaire ou le journal. On va donc trouver du côté des hommes des narrations présentant une vision globale de la famille et des rapports intrafamiliaux là où, du côté des femmes, on a un centrage sur le sujet et ses sentiments dans un contexte faisant intervenir un nombre plus limité de personnes : l'amant, l'amie, la mère. (Il s'agit là de deux grandes tendances, qui présentent évidemment des exceptions)

Les rapports filiaux s'inscrivent eux aussi dans des schémas quasi prédéterminés.

13 Du côté masculin, le schéma de base dans la littérature consiste en une rébellion du fils jeune et " moderne » contre un père traditionnel et tyrannique. Le père est celui qui empêche le fils d'être. Cette configuration s'est cristallisée dans la littérature sous la forme d'un conflit social (amour entre le fils de la famille et une domestique) ou d'un conflit oedipien (amour entre le fils et sa belle-mère ou la concubine du père).

Du côté féminin, on se trouve, en général, face à une autoreprésentation : jeune femme moderne, intellectuelle et citadine, qui se définit essentiellement par rapport à sa mère, dans un rapport qui peut apparaître tout aussi personnel que relevant d'un stéréotype.

\section{Le cas des écrivaines des années 1920}

Lorsqu'une " nouvelle littérature ", littérature en langue vernaculaire, émerge dans les années 1920 en Chine, quelques écrivaines prennent d'emblée leur place dans ce nouveau paysage littéraire. Parmi les premières, se trouvent Bing Xin 冰心(1900-1999), Lu Yin 盧隱 (1898-1934), Ling Shuhua 淩叔華 (1900-1990) et Feng Yuanjun 馮沅君(1900-1974). ${ }^{4}$ Chacune d'elles négocie et représente, à sa façon, en fonction de son histoire, un rapport particulier à la filiation dans son œuvre. Ce rapport évolue au fil du temps et la représentation du père n'est abordée ici que dans les toutes premières œuvres de ces écrivaines, avec pour objectif d'ouvrir un champ de réflexion par rapport à cette représentation, à partir de quelques textes, et non pas de présenter un travail 
exhaustif.

\section{Bing Xin 冰心 - ou le père selon la tradition}

Bing Xin commence à écrire très jeune. Dans sa première nouvelle, publiée en 1919, elle aborde la question de l'instruction des femmes et de son rôle dans les rapports de couple (« Liang ge jiating » 兩個家庭 [Deux familles]). Présente dans les cercles progressistes œuvrant pour une réforme de la littérature, elle en reprend les thèmes et ses premiers écrits reflètent les préoccupations générales de l'époque sous la forme de wenti xiaoshuo, nouvelles à thèse. ${ }^{5}$

Dans « Siren du qiaocui » 斯人独憔悴 (1919) [Seul, tu te ronges de chagrin] ${ }^{6}$, elle présente le conflit père traditionnel / fils modernes. Symbole d'un monde en voie de disparition, le père a pour activité principale et très sérieuse de jouer aux cartes avec ses amis. Personne n'ose le déranger lorsqu'une partie est en cours. En parallèle, il se montre tyrannique avec ses fils, leur reprochant de s'impliquer dans un combat politique, au détriment de leurs études, ce qu'il ne supporte pas, mais les empêchant finalement d'étudier en dépensant tout l'argent du foyer au jeu. Bing Xin axe le débat sur l'investissement politique au nom de la patrie, les fils voulant la sauver en tant que "Chinois », le père ne se posant même pas la question. Si l'image qui est donnée du père est négative, une figure paternelle positive est maintenue sous la forme de la patrie dont les jeunes gens se sentent fils. Un personnage féminin, sœur et fille, est présent du début à la fin de l'histoire. Témoin du conflit, des errances, elle intervient comme médiatrice, conseillère dans une position ambivalente entre, d'une part, un côté prescriptif - traditionnel - (ce qui se fait et ce qui ne se fait pas) et, de l'autre, un soutien silencieux à ses frères - représentant la modernité recherchée. Mais son rôle se limite à cela. Elle n'a pas de revendication propre, elle est juste là. Il n'y a pas d'issue à ce conflit : la nouvelle débute sur le conflit père/fils et se conclut sur la découverte par les fils qu'ils ne pourront faire leurs études. Le père est alors absent, les fils impuissants.

Bing Xin reprend ce conflit de génération dans «Qiu yu qiu feng chou sha ren » 秋雨秋風愁薢人 [Pluie et vent d'automne, mélancolie infinie], publié en 1919. ${ }^{7}$ Elle y présente une jeune fille cultivée acceptant un mariage arrangé avec un homme et une famille au style de vie ne lui correspondant pas. C'est autant par amour pour ses parents (père et mère) que par impossibilité d'échapper à son sort que la jeune fille accepte le mariage. Bing Xin se centre là sur le personnage féminin et son ressenti sans s'appesantir ni sur l'image paternelle ni sur l'image maternelle.

Quelques mois plus tard, dans “ Shi shei duansong le ni? 》 是誰斷送了你? [Qui t’a brisé ?] 1920, elle reprend le premier schéma avec le conflit de génération mais cette fois, le père s'oppose directement à sa fille. La jeune femme souhaite faire des études, ce que refuse son père. Il représente ici le monde d'autrefois, celui où le talent d'une femme était d'être sans vertu (女人 無才便是德 Nüren wu cai bian shi de). Pour faire céder sa fille, il lui coupe les vivres et elle en meurt de désespoir.

L'évolution qui se fait d'un texte à l'autre semble retracer l'évolution de Bing Xin par rapport à une problématique qu'elle semble observer de l'extérieur 
mais ne connait pas personnellement. Et si le père est désigné comme limitant ses enfants, un lien avec la tradition est maintenu au travers de différents éléments. Ainsi, par un curieux jeu à la fois inhabituel et audacieux, elle donne à ses deux premiers textes un titre extrait de poèmes des Tang, représentatifs donc de la société traditionnelle et, en même temps, ici, représentatif du malheur de jeunes gens victimes et en lutte contre cette société traditionnelle. Comme si elle avait voulu établir par ce biais une continuité avec le passé et une continuité positive puisqu'elle renvoie à une communauté de sentiments à un millénaire d'écart. Comme si, aussi, la culture tenait lieu de figure paternelle lorsque celle-ci est défaillante.

Dans le troisième texte, elle prend une position plus radicale avec un titre on ne peut plus explicite : Qui t'a brisée ? Sous-entendant pour qui t'es-tu sacrifiée ? Elle se fait là le porte parole des jeunes femmes opprimées de l'époque.

Finalement, Bing Xin semble hésitante par rapport à la représentation qu'elle souhaite donner du père et elle reste assez pondérée. Elle reprend l’image commune à l'époque du père traditionnel, bloquant ses enfants dans leur volonté d'émancipation mais, en même temps, elle fait une distinction entre l'homme et la culture qu'il représente, maintenant une image paternelle positive au travers de la culture et de la patrie. Alors qu'à l'époque, c'est autant le père que la culture qu'il représente qui sont rejetés par la jeunesse rebelle (masculine) en quête de modernité.

Sa position peut vraisemblablement être mise en rapport avec son expérience personnelle. Des textes qu'elle a consacrés plus tard à son père ressort un homme cultivé, peu présent mais souhaitant l'être, complice avec la mère face aux enfants ${ }^{8}$, une image peu en accord avec celle du père à abattre, despote familial, récurrente à l'époque.

\section{Lu Yin 盧隱 - ou le père inconnu}

Lu Yin s'illustre dans le domaine des lettres peu après Bing Xin et dans un réseau proche (celui des intellectuels pékinois) mais tant leur destin que leur rapport au monde et la représentation de la figure paternelle diffèrent grandement ${ }^{9}$.

La figure paternelle est présente mais brièvement dans « Haibin guren » 海 滨故人 [Amies du bord de mer] texte publié en 1923 qui consacre Lu Yin dans le monde des lettres. Apparaissant au tout début de l'histoire, le père est celui qui ne supporte pas les cris de l'enfant, or cet enfant est appelé à devenir le personnage principal de l'histoire et est considéré comme un double de l'auteure. Position initiale et incompréhension, le père fait figure d'inconnu. Lu Yin lui reconnait une place mais montre aussi qu'il ne l'occupe pas. Ensuite, son héroïne vit sa vie.

Si Lu Yin intitule l'un de ses textes « Fuqin » 父親 [Père] en 1925, le thème principal en est d'abord et surtout une histoire d'amour entre un jeune homme et la concubine de son père. Par la force des choses, quel qu'ait pu être le regard $\mathrm{du}$ jeune homme sur son père avant la rencontre avec la jeune femme, la figure paternelle se transforme, après la rencontre, en une caricature : considéré comme vieux (42 ans) abusant de l'opium et des femmes, lâche et menteur car il n'a obtenu la main de la jeune femme qu'en prétendant être veuf. Le père 
présenté ici n'en est pas un, c'est un homme de désirs, géniteur d'enfants dont il ne s'est pas occupé.

La place centrale revient, dans l'œuvre de Lu Yin, à la mère, idéalisée et absente. Une absence qui fait cruellement souffrir les jeunes héroïnes présentes dans les nouvelles de Lu Yin, au point, souvent, de leur coûter la vie. ${ }^{10}$

\section{Ling Shuhua 淩叔華 - culture, tradition et paternité}

Issue d'une famille représentative de la société traditionnelle, Ling Shuhua a consacré une grande partie de son œuvre à la description de son milieu d'origine et la filiation joue un rôle particulièrement important tant dans son histoire personnelle que dans son œuvre. ${ }^{11}$ Ainsi, elle retrace celle de sa mère, issue d'une famille de lettrés, enfant perdue, recueillie dans une famille l'aimant, puis retrouvée et rendue à sa famille d'origine. ${ }^{12}$ Du côté paternel, le lien s'est créé sur un plan culturel : elle dit devoir à son père son goût pour la peinture et la calligraphie. Dans les faits, c'est un ami de son père qui remarque ses dessins et suggère au père de lui faire donner des cours. ${ }^{13}$ Ce talent vaudra dès lors à Ling Shuhua une place particulière auprès de ce père, grand amateur de peinture traditionnelle.

Nombreux sont les textes - autobiographiques ou non - dans lesquels elle aborde la figure du père avec un regard d'enfant : homme lointain, représentant le pouvoir et l'autorité au sein de la famille, un pouvoir subi et douloureux pour les femmes, lointain et bienveillant pour les enfants, comme lorsque la petite fille assiste à l'arrivée de la sixième concubine dans la famille. ${ }^{14}$ Et c'est cette image qui domine. Ling Shuhua présente une figure paternelle puissante et potentiellement destructrice mais moins du fait de l'individu lui-même que de la société qui lui a donné cette place. Elle ne caricature pas les pères mais présente les conséquences de leurs actes, mettant en cause la société qui leur a donné ce pouvoir.

\section{Feng Yuanjun 馮沅君 - un père absent}

Feng Yuanjun a peu écrit et sur une période très brève mais ses textes ont profondément marqué le monde littéraire de l'époque pour leur audace, leur sincérité et une vraie présence. ${ }^{15}$

Si elle fait partie des quatre écrivaines reconnues de ce début des années 1920, elle fait ici figure à part dans la mesure où la figure du père est absente de ses écrits, la mère étant, elle, en revanche, au cœur de son écriture. ${ }^{16}$ Feng Yuanjun met en scène, de façon récurrente, le conflit intérieur d'une jeune femme, entre attachement pour sa mère et amour pour un homme. Et ce qui apparaît comme un choix impossible trouve des issues variées, assumées bien que jamais heureuses. ${ }^{17}$

Pour une jeune femme explorant sans détours les circonvolutions des sentiments de ses héroïnes, cette absence d'évocation peut relever tant d'une 
impossibilité de dire le père que d'un choix délibéré. Feng Yuanjun a effectivement perdu son père très jeune tandis que sa mère $a$, elle, été très présente assumant la prise en charge de Feng Yuanjun et ses frères aînés en travaillant comme directrice d'école. Et ce sont ses frères qui vont remplir un rôle protecteur en l'aidant lorsqu'elle veut faire des études supérieures.

Le père ou son image est, chez elle, simplement laissé de côté.

\section{Conclusion}

Ce qui ressort de ces quelques lectures, c'est avant tout le lien existant entre l'expérience personnelle des auteures et la représentation du père qu'elles proposent dans leur œuvre. Toujours peu présent dans leur vie, il peut apparaître sous différentes formes dans leur écriture : personnage imaginé chez Bing Xin, qui s'extrait de son histoire personnelle pour se fondre dans les stéréotypes de l'époque et produire un père faible, castrateur; personnage proche du modèle chez Ling Shuhua, plutôt indifférent mais capable d'un intérêt pour le talent de l'un de ses enfants ; lointain et sans lien positif possible chez Lu Yin.

La figure du père apparaît également en lien avec celle de l'identité culturelle, que ce soit parce que, le père défaillant, l’identité chinoise tient lieu de figure paternelle (Bing Xin) ou parce que le lien naît d'un élément culturel représentatif d'une identité chinoise traditionnelle (Ling Shuhua). La filiation qui met en jeu le père sur un plan individuel fait intervenir la culture au niveau de la société et un glissement s'opère d'un niveau à l'autre lorsque cela s'avère nécessaire. Si la représentation du père semble a priori manquer dans les œuvres féminines de ce début des années 1920, elle est néanmoins plus présente qu'il ne le semble de prime abord, au titre d'une filiation, à laquelle il est difficile d'échapper en tant qu'elle est constitutive de toute identité.

\section{Notes}

1 Sur la representation de la mère, voir Sally T. Lieberman, The Mother \& Narrative Politics in Modern China, Charlottesville and London, University Press of Virginia, 1998.

2 Ces quatre écrivaines sont celles présentes dans l'anthologie littéraire de référence de la période, qui contenant peu d'œuvres féminines, informe a minima sur leur statut dans le monde littéraire. V. Zhongguo xin wenxue daxi 1917-1927 中國新文學大系 1917-1927 [Anthologie de la nouvelle littérature chinoise 1917-1927], 10 tomes, dir. par Zhao Jiapi, rééd. Taipei, Yeqiang chubanshe, 1990 (1ère éd. 1935 -36). Pour un ensemble plus complet, récent et accessible en anglais, voir Amy D. Dooling \& Kristina M. Torgeson, Writing Women in Modern China - An Anthology of Women's Literature from the Early Twentieth Century, New York, Columbia University Press, 1998.

3 V. Liu Yuanyuan 劉媛媛, Ta shi jie - Xiandangdai Zhongguo nüxing wenxue tanxi 她 視界 - 現當代中國女性文學探析[Elle regarde le monde - Investigations sur la littérature féminine chinoise moderne et contemporaine], Taiyuan, Shanxi renmin chubanshe, 2010.

4 Sur l'écriture féminine en Chine au XXe siècle, outre les autres ouvrages ici mentionnés, voir, pour différents points de vue, Tani Barlow (éd.), Gender Politics in Modern China - Writing and Feminism, Durham \& London, Duke University Press, 1993; Rey Chow, Woman and Chinese Modernity - The Politics of Reading Between West and East, Minneapolis, University of Minnesota Press, 1991; Yan Chunde 閻純德, Ershi shiji Zhongguo nüzuojia yanjiu 二十世紀中國女作家研究 [Recherches sur les 
écrivaines chinoises du XXe siècle], Beijing, Beijing yuyan wenhua daxue chubanshe, 2000.

5 Pour une biographie détaillée, voir Chen Ru 陳恕, Bing Xin quan zhuan 冰心全傳 [Biographie complète de Bing Xin], Beijing, Zhongguo qingnian chubanshe, 2011. Sur son oeuvre, voir Jin Siyan, L'écriture féminine chinoise du XXe siècle à nos jours, Paris, You Feng, 2008, p. 69-74 ; Sally T. Lieberman, The Mother \& Narrative Politics in Modern China, Charlottesville and London, University Press of Virginia, 1998, p. 43-50.

6 Vers extrait d'un poème de Du Fu 杜甫 (712-770), 夢李白 « Meng Li Bai » [Rêvant à Li Bai].

7 Référence à un poème de Zhang Yue 張說 (667-730), 鄴都引 «Yedu yin » [Yedu].

8 V. par exemple, Bing Xin, 我的父親 «Wo de fuqin » [Mon père], in Guanyu nüren he nanren 關於女人和男人[A propos des hommes et des femmes], Guilin, Guangxi shifan daxue chubanshe, 2002, p. 294-296.

9 Lu Yin commence à publier en 1920 et s'implique activement dans le monde littéraire, en participant à la fondation de la Société de recherches littéraires (Wenxue yanjiu hui). Pour sa biographie et son oeuvre, voir Jin Siyan, L'écriture féminine chinoise du XXe siècle à nos jours, Paris, You Feng, 2008, p. 74-83; Jin Li 金理 (éd.) Lu Yin zizhuan 盧 隱自傳[Autobiographie de Lu Yin], Kunming, Yunnan renmin chubanshe, 2011 ; Fu Guangming 傅光明(éd.), Yi ge qingfu de riji 一個情婦的日記 [Journal d'une amante], Beijing, Jinghua chubanshe, 2006 ; Xiao Feng 肖鳳, Lu Yin pingzhuan 盧隱評傳 [Lu Yin : Biographie critique], Beijing, Zhongguo shehui chubanshe, 2007.

10 Née le jour où sa grand-mère maternelle est morte, Lu Yin a été rejetée par sa mère et confiée à une nourrice loin de chez elle. Son père décède alors qu'elle a 6 ans. Elle n'a jamais pu établir de rapport affectif avec sa mère et elle-même décède en mettant au monde sa deuxième fille.Sur sa représentation des femmes, voir Jin Feng, « Sentimental autobiographies: Feng Yuanjun, Lu Yin and the New Woman ", The New Woman in Early Twentieth-Century Chinese Fiction,West Lafayette, Purdue University Press, 2004, p. 126-148.

11 V. Su Hua (Ling Shuhua), Ancient Melodies, London, The Hogarth Press, 1969 (rééd.). Ce recueil, rédigé directement en anglais par Ling Shuhua avec le soutien de Virginia Woolf, est consacré à son enfance et sa famille. V. aussi Chen Xueyong 陳學勇, Gaomen juzu de lanhua - Ling Shuhua de yisheng 高門巨族的蘭花 - 淩叔華的一生 [Une orchidée de grande famille - La vie de Ling Shuhua], Beijing, Renmin wenxue chubanshe, 2010 ; Patricia Laurence, Lily Briscoe's Chinese Eyes - Bloomsbury, Modernism and China, Columbia, University of South Carolina Press, 2003 ; Jin Siyan, L'écriture féminine chinoise du XXe siècle à nos jours, Paris, You Feng, 2008, p. 88-104.

12 V. Su Hua « My mother's marriage », Ancient Melodies, p. 21-35.

13 V. Su Hua « My first lesson in painting », Ancient Melodies, p. 78-87.

14 《Yi jian xishi »—件喜事 [Un événement heureux] 1936, repris dans Su Hua sous le titre de « A happy event ", Ancient Melodies, p. 51-63.

15 Sur sa biographie, voir Xu Zhijue 許志傑, Lu Kanru he Feng Yuanjun 陸㑆如和馮沅 君 [Lu Kanru et Feng Yuanjun], Jinan, Shandong huabao chubanshe, 2006.

16 V. «Gejüe » 隔絕 [Séparation] 1923, “Gejüe zhi hou »隔絕之後 [Après la séparation] 1923, « Cimu » 慈母 [Une mère aimante] 1923.

17 Sur son oeuvre, voir Jin Feng, «Sentimental autobiographies: Feng Yuanjun, Lu Yin and the New Woman ", The New Woman in Early Twentieth-Century Chinese Fiction, West Lafayette, Purdue University Press, 2004, p. 126-148; Sally T. Lieberman, The Mother \& Narrative Politics in Modern China, Charlottesville and London, University Press of Virginia, 1998, p. 116-124.

\section{Pour citer cet article}

Référence électronique

Jacqueline ESTRAN, « Hist` ire d'une absence : la place du père dans les œuvres des écrivaines chin ises des années 1920. », Transtext(e)s Transcultures 跨文本跨文化 [En ligne], 8 | 2013, mis en ligne le 02 décembre 2013, c' nsulté le 15 janvier 2014. URL : 
Histoire d'une absence : la place du père dans les œuvres des écrivaines chinoises des années 1920.

http://transtexts.revues.' $r g / 496$

\section{Auteur}

\section{Jacqueline ESTRAN}

Maître de c' nférences en études chin` ises, Jacqueline Estran travaille sur l'hist ire s' ciale de la littérature (Poésie et liberté dans la Chine républicaine : la revue Xinyue, 1928-1933, Harrass` witz Vlg., 2010) et l'écriture féminine du XXe siècle (« Exemplaire 'u à part, le destin singulier de Lin Huiyin (1904-1955) », 2012 ; « Différence, distance et prise de c' nscience : Su Xuelin (1897-1999) et la France », 2012).

Maître de c’ nférences in Chinese Studies, Jacqueline Estran w’ rks ` $\mathrm{n}$ s`cial hist 'ry ` $\mathrm{f}$ literature (Poésie et liberté dans la Chine républicaine : la revue Xinyue, 1928-1933, Harrass ' witz Vlg., 2010) and is interessed in twentieth-century w` men's writing and related questi ns ("Exemplaire 'u à part, le destin singulier de Lin Huiyin (1904-1955) », 2012 ; « Différence, distance et prise de c’ nscience : Su Xuelin (1897-1999) et la France », 2012).

Articles du même auteur

Un monde rêvé : la France dans la revue Xinyue (1928-1933) [Texte intégral] Paru dans Transtext(e)s Transcultures 跨文本跨文化, 1 | 2006

\section{Droits d'auteur}

(C) T us dr` its réservés 